\title{
Synthesis of 2-(oxadiazolo, pyrimido, imidazolo, and benzimidazolo) substituted analogues of 1,4-benzodiazepin-5-carboxamides linked through a phenoxyl bridge
}

\author{
N KAUR* and D KISHORE \\ Department of Chemistry, Banasthali University, Banasthali-304022 (Rajasthan), India \\ e-mail: nvjithaans@gmail.com
}

MS received 27 September 2013; revised 3 April 2014; accepted 6 May 2014

\begin{abstract}
Exceedingly facile single-step expedient protocols based on the versatility and reactivity of corresponding intermediates amidine and imidate (8 and 9), derived from 5-carboxamido-1,4-benzodiazepin-5-(4'methylpiperazinyl)-carboxamide have been developed to provide an easy installation of the oxadiazole, pyrimidine, imidazole and benzimidazole (9-14) based privileged templates at 2-position of 5-carboxamido-1,4benzodiazepin-5-(4'-methylpiperazinyl)-carboxamide (4), through a phenoxyl spacer, by utilizing the synthetic strategy depicted in schemes 1 and 2 .
\end{abstract}

Keywords. 1,4-benzodiazepine; oxadiazole; pyrimidine; imidazole; benzimidazole; amidine and imidate ester.

\section{Introduction}

Recently, the privileged ${ }^{1}$ molecular framework of benzodiazepines has been actively studied in view of their ability to provide ligands to a number of functionally and structurally discrete biological receptors. ${ }^{2-5}$ The advent of anti-human immunodeficiency virus (HIV) activity in 1,4-benzodiazepine and imidazole derivative $\mathrm{TIBO}^{6}$ (a) (tetrahydroimidazobenzodiazepinone), the dipyrido diazepine derivative (nevirapine) ${ }^{7}$ (b), pyrimidine derivative (etravirine $)^{8}$ (c) and oxadiazole derivative (raltegravir) ${ }^{9}$ (d) (figure 1) prompted us to explore the possibility of developing some such analogues of 1,4-benzodiazepines which contained in its nucleus the vital fragments of oxadiazole, ${ }^{10}$ pyrimidine, ${ }^{11}$ imidazole, ${ }^{12}$ and benzimidazole ${ }^{13}$ scaffold on the premise that their presence in tandem with the same molecular framework could contribute significantly to provide a beneficial effect on the overall biological efficacy in the resulting molecules.

To create the novel heterocyclic scaffolds of biological interest through the simple and straightforward expedient routes, we explored the amidine ${ }^{14}(\mathbf{8})$ and imidate $\operatorname{ester}^{15}(\mathbf{9})$ based cyclization reactions for the incorporation of the oxadiazole, pyrimidine, imidazole and benzimidazole based privileged template on the 2position of 1,4-benzodiazepine nucleus (3) through a phenylamino spacer (schemes 1 and 2).

\footnotetext{
*For correspondence
}

\section{Experimental}

\subsection{General procedures}

All the melting points were determined in open glass capillaries and are uncorrected. The IR spectra were recorded on $\mathrm{KBr}$ disc using Perkin Elmer-1800 infrared. ${ }^{1} \mathrm{H}$ NMR and ${ }^{13} \mathrm{C}$ NMR spectra were recorded in $\mathrm{CDCl}_{3}$ on Brucker Avance $400 \mathrm{MHz}$ spectrometer with TMS as internal standard (chemical shifts are expressed in $\delta \mathrm{ppm}$ ). The mass spectra were recorded on a Joel SX-102 (FAB) mass spectrometer at $70 \mathrm{eV}$. The reactions were monitored by the TLC on silica gel G plates in the solvent system benzene-methanol mixture (9:1).

$N$-chloroacetylisatin (2) (M.p. $210-11^{\circ} \mathrm{C}$ ) and methyl1,3-dihydro-2 $H$-[1,4]-benzodiazepin-2-one-5-carboxylate (3) (M.p. $173-75^{\circ} \mathrm{C}$ ) were prepared according to the reported procedure for their preparation in the literature. ${ }^{23-26}$

\subsection{Preparation of 1,3-dihydro-[2H]-[1,4]-} benzodiazepin-5-(4'-methylpiperazinyl)-carboxamide (4)

Methyl-1,3-dihydro-[2H]-[1,4]-benzodiazepin-2-one5-carboxylate (3) $(10.9 \mathrm{~g}, 0.05 \mathrm{~mol})$ and $\mathrm{N}$-methyl piperazine $(5.0 \mathrm{~g}, 0.05 \mathrm{~mol})$ were taken in ethanol $(100 \mathrm{~mL})$. The reaction mixture was refluxed for $12 \mathrm{~h}$ on the water bath. The completion of the reaction was checked by TLC. The mixture was cooled and poured 
<smiles>CC(C)=CCN1Cc2cc(Cl)cc3[nH]c(=O)n(c23)CC1C</smiles>

a<smiles>Cc1ccnc2c1NC(=O)c1cccnc1N2C1CC1</smiles>

b<smiles>N#Cc1ccc(Nc2nc(N)cc(Oc3ccc(C#N)cc3)n2)cc1</smiles>

c<smiles>Cc1nnc(C(=O)NC(C)(C)c2nc(C(=O)NCc3ccc(F)cc3)c(O)c(=O)n2C)o1</smiles>

d

Figure 1. Structures of TIBO (a), (nevirapine) (b), (etravirine) (c) and (raltegravir).

on crushed ice, the resulting solid was filtered, washed with dilute ethanol, dried and recrystallized from ethanol-chloroform mixture (1:9), to give compound 4 (12.37 g, $75 \%$, M.p. $\left.257-258^{\circ} \mathrm{C}\right)$. IR (KBr) $\mathrm{cm}^{-1}: 3330$ (NH str.), 2950 (C-H str. ArH), 1675 (C=O str.), 1660 $(\mathrm{C}=\mathrm{C}$ str. $\mathrm{ArH}), 1590(\mathrm{C}=\mathrm{N}$ str. $), 1580$ (NH bending), 1430 (C-H bending, $\left.\mathrm{CH}_{3}\right), 1140$ (C-N str.); ${ }^{1} \mathrm{H}$
NMR (400 MHz, $\left.\mathrm{CDCl}_{3}\right) \delta$ ppm: $8.0(\mathrm{~s}, 1 \mathrm{H}), 7.27-7.86$ (m, 4H), 3.60 (s, 2H), $3.20(\mathrm{t}, J=7.4 \mathrm{~Hz}, 4 \mathrm{H}), 2.27$ $(\mathrm{t}, J=7.2 \mathrm{~Hz}, 4 \mathrm{H}), 2.23(\mathrm{~s}, 3 \mathrm{H}) ;{ }^{13} \mathrm{C} \mathrm{NMR}(400 \mathrm{MHz}$, $\left.\mathrm{CDCl}_{3}\right) \delta$ ppm: $45.2\left(\mathrm{CH}_{3}\right), 47.3\left(\mathrm{CH}_{2}\right.$, diazepine $), 49.5$, $82.3\left(\mathrm{CH}_{2}\right.$, piperazine), 113.0, $117.7(\mathrm{C}$, arene $), 125.4$, 116.7 (C, arene), 128.2, $126.5(\mathrm{CH}$, arene), 149.5, $158.2(\mathrm{C}=\mathrm{N}), 164.5(\mathrm{C}=\mathrm{O})$; $\mathrm{MS}:\left[\mathrm{M}^{+}\right]$: 286, Anal.<smiles>O=C1Nc2ccccc2C1=O</smiles>
1<smiles>O=C1C(=O)N(C(=O)Cl)c2ccccc21</smiles>
2<smiles>COC(=O)C1=NCC(=O)Nc2ccccc21</smiles><smiles>C[C@H]1CNCCN1</smiles>
3<smiles>CN1CCN(C(=O)C2=NCC(=O)Nc3ccccc32)CC1</smiles>

4<smiles>[R1]C(=O)C1=NCC(Cl)=Nc2ccc(CCCCC)cc21</smiles><smiles>[R]C(=O)C1=NCC(Oc2ccc(C#C)cc2)=Nc2ccccc21</smiles><smiles>[R]C(=O)C1=NCC(Oc2ccc(C(=[Ni])OC(C)C)cc2)=Nc2ccccc21</smiles><smiles>CCOC(=O)c1nc(-c2ccc(OC3=Nc4ccccc4C(C(=O)CC)=NC3)cc2)nc(O)c1O</smiles><smiles>[Z17]C(=O)C1=NCC(Oc2ccc(/C(N)=N/O)cc2)=Nc2ccccc21</smiles>

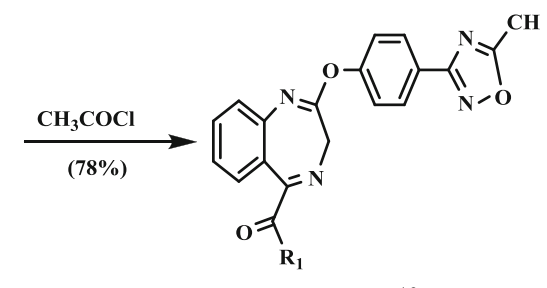

Scheme 1. Synthesis of oxadiazole and pyrimidine bearing benzodiazepine nucleus. 


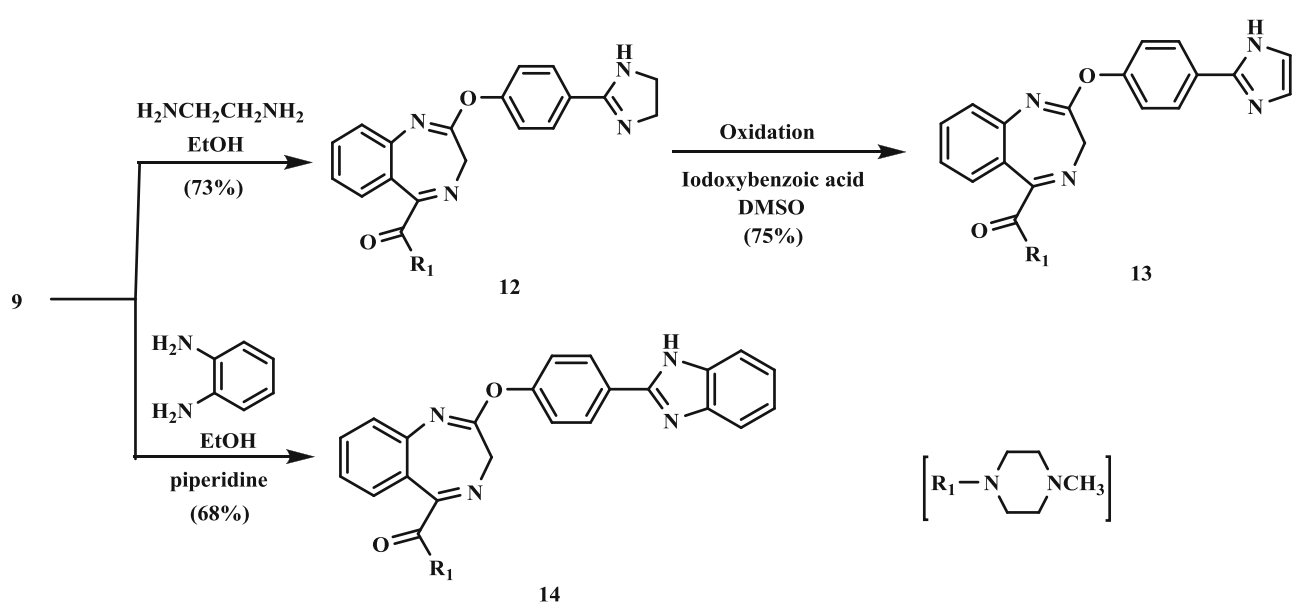

Scheme 2. Synthesis of imidazole and benzimidazole bearing benzodiazepine nucleus.

calcd. for $\mathrm{C}_{15} \mathrm{H}_{18} \mathrm{~N}_{4} \mathrm{O}_{2}$ : C, 62.92; H, 6.34; N, 19.57 . Found: C, 62.75; H, 6.32; N, 19.48.

\subsection{Preparation of 2-chloro-[1,4]-benzodiazepin-5- (4'-methylpiperazinyl)-carboxamide (5)}

A solution of compound $4(10 \mathrm{~g}, 0.06 \mathrm{~mol}), \mathrm{POCl}_{3}$ $(5 \mathrm{~mL}, \quad 0.06 \mathrm{~mol}), \quad N, N$-dimethylaniline $\quad(14 \mathrm{~mL}$, $0.1 \mathrm{~mol})$, and benzene $(100 \mathrm{~mL})$ were refluxed for $7 \mathrm{~h}$ and allowed to cool overnight. The cold reaction mixture was poured in ice water $(100 \mathrm{~mL})$ and stirred for $30 \mathrm{~min}$. until the reaction mixture reached room temperature. It was then extracted with ether and the solvent layer was washed with water and brine, dried (over anhydrous $\mathrm{MgSO}_{4}$ ), filtered and evaporated. Trituration with ether gave compound $5(8.0 \mathrm{~g}, 75 \%$,

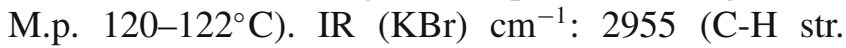
$\mathrm{ArH}), 1680(\mathrm{C}=\mathrm{O}$ str. $), 1590(\mathrm{C}=\mathrm{C}$ str. $\mathrm{ArH}), 1570$ ( $\mathrm{C}=\mathrm{N}$ str.), 1435 (C-H bending, $\left.\mathrm{CH}_{3}\right), 1140$ (C-N str.), 710 (C-Cl str.); ${ }^{1} \mathrm{H}$ NMR (400 MHz, $\left.\mathrm{CDCl}_{3}\right) \delta$ ppm: $7.33-7.83(\mathrm{~m}, 4 \mathrm{H}), 3.60(\mathrm{~s}, 2 \mathrm{H}), 3.20(\mathrm{t}, J=7.4 \mathrm{~Hz}$, $4 \mathrm{H}), 2.27(\mathrm{t}, J=7.5 \mathrm{~Hz}, 4 \mathrm{H}), 2.26(\mathrm{~s}, 3 \mathrm{H}) ;{ }^{13} \mathrm{C} \mathrm{NMR}$ $\left(400 \mathrm{MHz}, \mathrm{CDCl}_{3}\right) \delta$ ppm: $45.2\left(\mathrm{CH}_{3}\right), 46.0\left(\mathrm{CH}_{2}\right.$, diazepine), 47.8, $95.3\left(\mathrm{CH}_{2}\right.$, piperazine $), 114.6,118.8$ (C, arene), 126.5, $128.2(\mathrm{CH}$, arene), 148.6, 155.1, $(\mathrm{C}=\mathrm{N}) 158.3(\mathrm{C}-\mathrm{Cl}), 164.6(\mathrm{C}=\mathrm{O})$; MS: $\left[\mathrm{M}^{+}\right]$: 304, Anal. calcd. for $\mathrm{C}_{15} \mathrm{H}_{17} \mathrm{ClN}_{4} \mathrm{O}$ : C, 59.24; H, 5.65; N, 18.34. Found: C, 59.11; H, 5.67; N, 18.28.

\subsection{Preparation of 2-[4'-cyanophenoxyl-[1,4]- benzodiazepin-5-(-4"-methylpiperazinyl)]- carboxamide (7)}

To a solution of compound $5(1.50 \mathrm{~g}, 0.01 \mathrm{~mol})$ and 4-hydroxybenzonitrile (6) $(0.60 \mathrm{~g}, 0.01 \mathrm{~mol})$ in
$N$-methylpyrrolidone $(7.5 \mathrm{~mL})$ at $0-5^{\circ} \mathrm{C}$ was added potassium tert-butoxide $(1.14 \mathrm{~g}, 0.01 \mathrm{~mol})$ over a period of $6 \mathrm{~h}$. The reaction was allowed to reach to room temperature and then cold water $(300 \mathrm{~mL})$ was added. The reaction mixture was filtered; the residue was suspended in water $(150 \mathrm{~mL})$ and acidified to $\mathrm{pH}$ 6-7 using conc. $\mathrm{HCl}$. The product was filtered and washed with $15 \mathrm{~mL}$ of water. It was extracted by ethyl acetate $(2 \times 50 \mathrm{~mL})$. The product obtained on evaporation of solvent was washed with $5 \mathrm{~mL}$ of chilled ethyl acetate. It was finally dried at $55-60^{\circ} \mathrm{C}$ under vacuum to give compound 7 (1.26 g, 66\%, M.p. $\left.297-298.5^{\circ} \mathrm{C}\right)$. IR (KBr) cm ${ }^{-1}$ : 2980 (C-H str. ArH), 2225 (CN str.), $1685(\mathrm{C}=\mathrm{O}$ str. $), 1590(\mathrm{C}=\mathrm{C}$ str. $\mathrm{ArH}), 1525(\mathrm{C}=\mathrm{N}$ str.), 1470 (C-H bending, $\mathrm{CH}_{3}$ ), 1250 (C-O str.), 1035 (C-N str.); ${ }^{1} \mathrm{H}$ NMR $\left(400 \mathrm{MHz}, \mathrm{CDCl}_{3}\right) \delta$ ppm: $7.33-$ $7.81(\mathrm{~m}, 4 \mathrm{H}), 3.6(\mathrm{~s}, 2 \mathrm{H}), 3.20(\mathrm{t}, J=7.5 \mathrm{~Hz}, 4 \mathrm{H}), 2.27$ $(\mathrm{t}, J=7.6 \mathrm{~Hz}, 4 \mathrm{H}), 2.26(\mathrm{~s}, 3 \mathrm{H}) ;{ }^{13} \mathrm{C}$ NMR $(400 \mathrm{MHz}$, $\left.\mathrm{CDCl}_{3}\right) \delta$ ppm: $42.1\left(\mathrm{CH}_{3}\right), 44.3\left(\mathrm{CH}_{2}\right.$, diazepine $), 45.6$, $58.5\left(\mathrm{CH}_{2}\right.$, piperazine), 106.0 (C-CN, cyanophenoxyl), $116.4(\mathrm{CH}$, cyanophenoxyl), 117.8, 148.4 (C, arene), $118.1(\mathrm{CN}), 127.5,131.2(\mathrm{CH}$, arene $), 134.1(\mathrm{CH}$, cyanophenoxyl), 164.5 (C-O, cyanophenoxyl), 165.7 $(\mathrm{C}=\mathrm{N}), 200.1(\mathrm{C}=\mathrm{O})$; $\mathrm{MS}:\left[\mathrm{M}^{+}\right]$: 387, Anal. Calcd. for $\mathrm{C}_{23} \mathrm{H}_{24} \mathrm{~N}_{5} \mathrm{O}_{2}$ : C, 68.64; H, 6.01; N, 17.40; Found: C, 68.47; H, 5.99; N, 17.35 .

\subsection{Preparation of 5-oxo-[(benzo-[1,4]-diazepin-2-} yloxy)-carboximidamide-5-(-4"-methylpiperazinyl)]carboxamide (8)

Hydroxylamine hydrochloride $(1 \mathrm{~g}, 0.01 \mathrm{~mol})$ in methanol $(10 \mathrm{~mL})$ was added to an equimolar stirred solution of potassium hydroxide in methanol $(10 \mathrm{~mL})$. The mixture was stirred for $15 \mathrm{~min}$ and the precipitated potassium chloride was removed by filtration. The 
filtrate was added to an equimolar amount of the above nitrile (7) $(3.87 \mathrm{~g}, \mathrm{~mol})$, and the solution was stirred overnight at $40^{\circ} \mathrm{C}$, then cooled to room temperature and concentrated. The resulting residue was triturated with water giving, after drying under vacuum, a white solid consisting mainly of the title product $8,(3.56 \mathrm{~g}, 85 \%$, M.p. $\left.181-83^{\circ} \mathrm{C}\right)$. IR $(\mathrm{KBr}) \mathrm{cm}^{-1}$ : 2600-3510 [broad $\mathrm{OH}$ str. (oxime)], 3310 [NH str. $\left(\mathrm{NH}_{2}\right.$ group)], 2855 (C-H str. ArH), $1690(\mathrm{C}=\mathrm{O}), 1635$ ( $\mathrm{NH}$ bending), 1615 $(\mathrm{C}=\mathrm{C}$ str. $), 1580(\mathrm{C}=\mathrm{N}$ str. $), 1530(\mathrm{C}=\mathrm{C}$ str. $\mathrm{ArH})$, 1480 (C-H bending $\mathrm{CH}_{3}$ ), 1170 (C-N str.), 1080 (C-O str.); ${ }^{1} \mathrm{H}$ NMR $\left(400 \mathrm{MHz}, \mathrm{CDCl}_{3}\right) \delta$ ppm: 7.33-7.78 (m, 4H), $7.19(\mathrm{~s}, 2 \mathrm{H}), 7.09-7.41(\mathrm{~m}, 4 \mathrm{H}), 3.6(\mathrm{~s}, 2 \mathrm{H})$, $3.20(\mathrm{t}, J=7.5 \mathrm{~Hz}, 4 \mathrm{H}), 2.28(\mathrm{t}, J=7.6 \mathrm{~Hz}, 4 \mathrm{H}), 2.25$ (s, 3H), $2.33(\mathrm{~s}, 1 \mathrm{H}) ;{ }^{13} \mathrm{C} \mathrm{NMR}\left(400 \mathrm{MHz}, \mathrm{CDCl}_{3}\right)$ $\delta$ ppm: $13.2(\mathrm{C}=\mathrm{N}-\mathrm{OH}), 45.4\left(\mathrm{CH}_{3}\right), 47.5\left(\mathrm{CH}_{2}\right.$, diazepine), 49.1, $118.0\left(\mathrm{CH}_{2}\right.$, piperazine $), 127.6,129.8$ ( $\mathrm{CH}$, phenoxyl), 135.5, 138.7, 146.6, $147.8(\mathrm{CH}$, arene), 143.3, 149.5 (C, arene), 134.0, 153.4 (C, phenoxyl), $157.6(\mathrm{C}=\mathrm{O}), 159.8(\mathrm{C}-\mathrm{O}), 164.5(\mathrm{C}=\mathrm{N})$; MS: $\left[\mathrm{M}^{+}\right]$: 420, Anal. Calcd. for $\mathrm{C}_{22} \mathrm{H}_{24} \mathrm{~N}_{6} \mathrm{O}_{3}$ : C, 62.84; $\mathrm{H}, 5.75$; N, 19.99; Found: C, 62.91; H, 5.76; N, 19.94.

\subsection{Preparation of 5-oxo-[(benzo-[1,4]-diazepin-2- yloxy)-ethylimidate-5-(-4"-methylpiperazinyl)]- carboxamide (9)}

The compound $7(3.87 \mathrm{~g}, 0.01 \mathrm{~mol})$ was dissolved in absolute ethanol $(10 \mathrm{~mL})$ and stirred for $15 \mathrm{~min}$ with ice-bath cooling. Then the imidate hydrochloride (9) was prepared by passing $\mathrm{HCl}$ gas through the above solution. $\mathrm{HCl}$ gas was passed for $7 \mathrm{~h}$ with constant stirring at room temperature. The completion of the reaction was checked by TLC, after completion the precipitation of reaction mixture was done by adding diethyl ether to it, filtered, dried over vacuum, and recrystallized from petroleum ether to yield compound 9, (2.68 g, 64\%, M.p. $\left.81-83^{\circ} \mathrm{C}\right)$. IR $(\mathrm{KBr}) \mathrm{cm}^{-1}: 3350$ (NH str.), 2950 (C-H str. ArH), 1670 (free $\mathrm{C}=\mathrm{O}$ str.), 1590 ( $\mathrm{NH}$ bending), 1595-1610 ( $\mathrm{C}=\mathrm{N}$ str.), 1555 $(\mathrm{C}=\mathrm{C}$ str. $\mathrm{ArH}), 1440\left(\mathrm{C}-\mathrm{H}\right.$ bending $\left.\mathrm{CH}_{3}\right), 1160(\mathrm{C}-$ $\mathrm{N}$ str.), 1140 (C-O str.), 1070 (C-O str.); ${ }^{1} \mathrm{H}$ NMR $\left(400 \mathrm{MHz}, \mathrm{CDCl}_{3}\right) \delta$ ppm: 7.33-7.83 (m, 4H), 6.35$7.20(\mathrm{~m}, 4 \mathrm{H}), 3.7(\mathrm{~s}, 1 \mathrm{H}), 3.6(\mathrm{~s}, 2 \mathrm{H}), 3.40(\mathrm{~s}, 3 \mathrm{H})$, $3.21(\mathrm{t}, J=7.4 \mathrm{~Hz}, 4 \mathrm{H}), 2.27(\mathrm{t}, J=7.5 \mathrm{~Hz}$, $4 \mathrm{H}), 2.23$ (s, 3H); ${ }^{13} \mathrm{C} \mathrm{NMR}\left(400 \mathrm{MHz}, \mathrm{CDCl}_{3}\right) \delta \mathrm{ppm}$ : $45.2\left(\mathrm{CH}_{3}\right), 47.4\left(\mathrm{CH}_{2}\right.$, diazepine $), 48.2,116.6\left(\mathrm{CH}_{2}\right.$, piperazine), 128.1, $127.0(\mathrm{CH}$, phenoxyl), 135.2, 129.3, $130.9,132.8(\mathrm{CH}$, arene), 148.1, 158.3 (C, arene), 134.1, 154.1 (C, phenoxyl), $156.6(\mathrm{C}=\mathrm{O}), 164.3(\mathrm{C}-$ O), $174.5(\mathrm{C}=\mathrm{N})$; MS: $\left[\mathrm{M}^{+}\right]$: 419, Anal. Calcd. for $\mathrm{C}_{23} \mathrm{H}_{25} \mathrm{~N}_{5} \mathrm{O}_{3}$ : C, 65.85; H, 6.01; N, 16.70; Found: C, $65.69 ; \mathrm{H}, 5.98 ; \mathrm{N}, 16.65$.
2.7 Preparation of 2-[4-(5-methyl-1,2,4-oxadiazol-3phenoxyl)-[1,4]-benzodiazepin-5-(4"'-methylpiperazinyl)]-carboxamide (10)

Compound 8 (2.10 g, $0.005 \mathrm{~mol})$ was refluxed in acetyl chloride $(10 \mathrm{~mL})$ for $5 \mathrm{~h}$. The solvent was evaporated under vacuum and the crude product was collected and crystallized from ethanol gave the product 10, (1.734 g, 78\%, M.p. $\left.118-20^{\circ} \mathrm{C}\right)$. IR (KBr) $\mathrm{cm}^{-1}: 2880$ (C-H str. ArH), $1680(\mathrm{C}=\mathrm{O}), 1610(\mathrm{C}=\mathrm{C}$ str. $), 1580$ ( $\mathrm{C}=\mathrm{N}$ str.), $1535(\mathrm{C}=\mathrm{C}$ str. $\mathrm{ArH}), 1430$ (C-H bending $\mathrm{CH}_{3}$ ), 1180 (C-N str.), 1090 (C-O str.); ${ }^{1} \mathrm{H}$ NMR $\left(400 \mathrm{MHz}, \mathrm{CDCl}_{3}\right) \delta$ ppm: 7.33-7.78 (m, 4H), 6.81$7.91(\mathrm{~m}, 4 \mathrm{H}), 3.6(\mathrm{~s}, 2 \mathrm{H}), 3.20(\mathrm{t}, J=7.3 \mathrm{~Hz}, 4 \mathrm{H})$, $2.60(\mathrm{~s}, 3 \mathrm{H}), 2.28(\mathrm{t}, J=7.4 \mathrm{~Hz}, 4 \mathrm{H}), 2.25(\mathrm{~s}, 3 \mathrm{H})$; ${ }^{13} \mathrm{C}$ NMR $\left(400 \mathrm{MHz}, \mathrm{CDCl}_{3}\right) \delta$ ppm: $12.4\left(\mathrm{CH}_{3}\right.$, oxadiazole), $44.0\left(\mathrm{CH}_{3}\right), 45.8\left(\mathrm{CH}_{2}\right.$, diazepine $), 47.2,49.4$ $\left(\mathrm{CH}_{2}\right.$, piperazine $), 117.5,127.4(\mathrm{CH}$, phenoxyl $), 131.6$, 135.8, 138.1 (CH, arene), 143.3, 146.5 (C, arene), 134.7, $153.5(\mathrm{C}$, phenoxyl), $158.7(\mathrm{C}=\mathrm{O}), 157.0$ (CO), $164.6(\mathrm{C}=\mathrm{N}), 168.4,175.1$ (2C, oxadiazole); MS: $\left[\mathrm{M}^{+}\right]$: 444, Anal. Calcd. for $\mathrm{C}_{24} \mathrm{H}_{24} \mathrm{~N}_{6} \mathrm{O}_{3}$ : C, 64.85; $\mathrm{H}$, 5.44; N, 18.91; Found: C, 64.68; H, 5.43; N, 18.96.

2.8 Preparation of 2-[4'-(4"-ethylcarboxylate-5",6"dihydroxypyrimidin-2"-yl)phenoxyl-[1,4]-benzodiazepin-5-(4"'-methyl-piperazinyl)-carboxamide (11) Compound 8

$(2.94 \mathrm{~g}, \quad 0.007 \mathrm{~mol})$ was suspended in chloroform $(20 \mathrm{~mL})$ and refluxed overnight in the presence of 1.0 equiv of dimethyl acetylenedicarboxylate $(0.87 \mathrm{~mL})$. After being cooled to room temperature, volatiles were evaporated and the residue was refluxed in xylene $(25 \mathrm{~mL})$ for $3 \mathrm{~h}$. The mixture was cooled to room temperature to allow the formation of precipitate of compound 11, was collected by filtration and washed with diethyl ether, $\left(0.645 \mathrm{~g}, 20 \%, 125-27^{\circ} \mathrm{C}\right)$. IR $(\mathrm{KBr})$ $\mathrm{cm}^{-1}$ : 3480-3500 (OH str.), 3010 (C-H str. ArH), 1685 $(\mathrm{C}=\mathrm{O}$ str. $), 1740(\mathrm{C}=\mathrm{O}$ str. ester $), 1640(\mathrm{C}=\mathrm{N}$ str. $)$, $1610(\mathrm{C}=\mathrm{C}$ unsaturated $), 1580(\mathrm{C}=\mathrm{C}$ str. $\mathrm{ArH}), 1460$ (C-H bending $\mathrm{CH}_{3}$ ), 1190 (C-N str.), 1150-1200 (ester group), 1090-1105 (C-O str.); ${ }^{1} \mathrm{H}$ NMR (400 MHz, $\left.\mathrm{CDCl}_{3}\right) \delta$ ppm: $12.20(\mathrm{~s}, 1 \mathrm{H}), 7.33-7.83(\mathrm{~m}, 4 \mathrm{H}), 6.83-$ $7.80(\mathrm{~m}, 4 \mathrm{H}), 5.35(\mathrm{~s}, 1 \mathrm{H}), 3.6(\mathrm{~s}, 2 \mathrm{H}), 4.30(\mathrm{q}, J=7.3$ $\mathrm{Hz}, 2 \mathrm{H}), 1.29(\mathrm{t}, J=7.4 \mathrm{~Hz}, 3 \mathrm{H}), 3.20(\mathrm{t}, J=7.5$ $\mathrm{Hz}, 4 \mathrm{H}), 2.27(\mathrm{t}, J=7.6 \mathrm{~Hz}, 4 \mathrm{H}), 2.26(\mathrm{~s}, 3 \mathrm{H}) ;{ }^{13} \mathrm{C}$ NMR $\left(400 \mathrm{MHz}, \mathrm{CDCl}_{3}\right) \delta$ ppm: $16.1\left(\mathrm{CH}_{3}\right.$, ester), $45.2\left(\mathrm{CH}_{3}\right), 47.0\left(\mathrm{CH}_{2}\right.$, diazepine $), 48.9,49.9\left(\mathrm{CH}_{2}\right.$, piperazine), $72.1\left(\mathrm{CH}_{2}\right.$, ester), 116.6, $130.3(\mathrm{CH}$, phenoxyl), 127.5, 155.8 (C, phenoxyl), 126.3, $130.6(\mathrm{CH}$, arene), 132.8, 148.2 (C, arene), 143.1, 145.4, 163.1 (C, pyrimidine), $149.8(\mathrm{C}-\mathrm{OH}$, pyrimidine $), 158.1(\mathrm{C}=\mathrm{O})$, 
$164.3(\mathrm{C}=\mathrm{N}, \mathrm{C}-\mathrm{O}), 168.1(\mathrm{C}=\mathrm{O}$, ester $) ; \mathrm{MS}:\left[\mathrm{M}^{+}\right]$: 544, Anal. Calcd. for $\mathrm{C}_{28} \mathrm{H}_{28} \mathrm{~N}_{6} \mathrm{O}_{6}: \mathrm{C}, 61.76 ; \mathrm{H}, 5.16$; N, 15.43; Found: C, 61.58; H, 5.14; N, 15.38.

\subsection{Preparation of 2-[4'-(dihydroimidazol-2"-} yl)phenoxyl-[1,4]-benzodiazepin-5-(4"-methylpiperazinyl)]-carboxamide (12)

A mixture ethylenediamine $(1.08 \mathrm{~g}, 0.01 \mathrm{~mol})$, imidate ester (9) $(0.586 \mathrm{~g}, 0.0014 \mathrm{~mol})$ and ethanol $(15 \mathrm{~mL})$ was refluxed for $11 \mathrm{~h}$. The solvent was distilled under reduced pressure and the residue was quenched in crushed ice. It was extracted with chloroform, washed with water, and dried over anhydrous sodium sulfate to give compound 12, $\left(0.44 \mathrm{~g}, 73 \%\right.$, M.p. $\left.136-37^{\circ} \mathrm{C}\right)$.

\subsection{Preparation of 2-[4'-(imidazol-2"'-yl)phenoxyl-} [1,4]-benzodiazepin-5-(4"'-methyl-piperazinyl)]carboxamide (13) via oxidation of compound 12

Iodoxybenzoic acid $(0.4 \mathrm{~g}, 1.53 \mathrm{mmol})$ was added as a solid to a solution of compound $12(0.0015 \mathrm{~mol})$ in DMSO $(1 \mathrm{~mL})$ and stirred at $45^{\circ} \mathrm{C}$ for $11 \mathrm{~h}$. The mixture was cooled to room temperature and quenched by addition of saturated aqueous $\mathrm{Na}_{2} \mathrm{~S}_{2} \mathrm{O}_{3}(1 \mathrm{~mL})$ and then basified with saturated aqueous $\mathrm{NaHCO}_{3}(1 \mathrm{~mL})$. Following extraction with EtOAc $(15 \mathrm{~mL})$, the organic phase was washed with water $(10 \mathrm{~mL})$ and brine $(10 \mathrm{~mL})$, dried $\left(\mathrm{MgSO}_{4}\right)$, and concentrated to yield the desired product $13,\left(0.481\right.$ g, $75 \%$, M.p. $\left.130-32^{\circ} \mathrm{C}\right)$. IR $(\mathrm{KBr}) \mathrm{cm}^{-1}: 3320(\mathrm{NH}$ str. imidazole ring), $2970(\mathrm{C}-\mathrm{H}$ str. $\mathrm{ArH}), 1680(\mathrm{C}=\mathrm{O}), 1680(\mathrm{C}=\mathrm{C}$ unsaturated $), 1625$ $(\mathrm{C}=\mathrm{N}$ str. $), 1590(\mathrm{NH}$ bending $), 1550(\mathrm{C}=\mathrm{C}$ str. $\mathrm{ArH})$, 1460 (C-H bending $\mathrm{CH}_{3}$ ), 1050 (C-N str.), 1040 (C-O str.); ${ }^{1} \mathrm{H}$ NMR $\left(400 \mathrm{MHz}, \mathrm{CDCl}_{3}\right) \delta$ ppm: $13.4(\mathrm{~s}, 1 \mathrm{H})$, $7.33-7.83(\mathrm{~m}, 4 \mathrm{H}), 7.02(\mathrm{~d}, J=7.8 \mathrm{~Hz}, 2 \mathrm{H}), 6.81-7.90$ $(\mathrm{m}, 4 \mathrm{H}), 3.6(\mathrm{~s}, 2 \mathrm{H}), 3.21(\mathrm{t}, J=7.4 \mathrm{~Hz}, 4 \mathrm{H}), 2.27(\mathrm{t}$, $J=7.3 \mathrm{~Hz}, 4 \mathrm{H}), 2.23(\mathrm{~s}, 3 \mathrm{H}) ;{ }^{13} \mathrm{C} \mathrm{NMR}(400 \mathrm{MHz}$, $\left.\mathrm{CDCl}_{3}\right) \delta$ ppm: $45.2\left(\mathrm{CH}_{3}\right), 47.1\left(\mathrm{CH}_{2}\right.$, diazepine $), 48.8$ $\left(\mathrm{CH}_{2}\right.$, piperazine $), 116.1,127.1(\mathrm{CH}$, phenoxyl), 122.5 (CH, imidazole), 126.5, $132.2(\mathrm{CH}$, arene), 130.3, 147.3 (C, arene), 132.5, 155.3 (C, phenoxyl), 148.7 (C, imidazole), $158.1(\mathrm{C}=\mathrm{O}), 164.4(\mathrm{C}=\mathrm{N}, \mathrm{C}-\mathrm{O})$; $\mathrm{MS}:\left[\mathrm{M}^{+}\right]$: 428, Anal. Calcd. for $\mathrm{C}_{24} \mathrm{H}_{24} \mathrm{~N}_{6} \mathrm{O}: \mathrm{C}, 67.27 ; \mathrm{H}, 5.65 ; \mathrm{N}$, 19.61; Found: C, 67.11; H, 5.63; N, 19.53.

\subsection{Preparation of 2-[4'-(benzimidazol-2"'-} yl)phenoxyl-[1,4]-benzodiazepin-5-(4"-methylpiperazinyl)]-carboxamide (14)

To a solution of imidate ester (9) (1.257 g, $0.003 \mathrm{~mol})$ in ethanol $(10 \mathrm{~mL})$ was added $2-3$ drops of piperidine and $o$-phenylenediamine $(1.08 \mathrm{~g}, 0.01 \mathrm{~mol})$. The mixture was heated under reflux for $11 \mathrm{~h}$ and then $1 \mathrm{~mL}$ of $\mathrm{AcOH}$ was added. The refluxing was continued for $1 \mathrm{~h}$. About half of the solvent was distilled off and the resulting mixture was allowed to stand at room temperature. The crystalline compound $\mathbf{1 4}$ thus separated was filtered, washed with $5 \mathrm{~mL}$ of cold aq. ethanol (50:50 by v/v) and dried, $\left(0.975 \mathrm{~g}, 68 \%\right.$, M.p. $\left.177-79^{\circ} \mathrm{C}\right)$. IR $(\mathrm{KBr}) \mathrm{cm}^{-1}: 3370(\mathrm{NH}$ str. benzimidazole ring), 3010 $(\mathrm{C}-\mathrm{H}$ str. $\mathrm{ArH}), 1670$ (free $\mathrm{C}=\mathrm{O}$ str.), $1610(\mathrm{C}=\mathrm{C}$ str. ArH), 1595 (C=N str.), 1560 (NH bending), 1440 (C$\mathrm{H}$ bending $\mathrm{CH}_{3}$ ), 1150 (C- $\mathrm{N}$ str.), 1110 (C-O str.); ${ }^{1} \mathrm{H}$ NMR (400 MHz, $\left.\mathrm{CDCl}_{3}\right) \delta$ ppm: 7.33-7.83 (m, 4H), 7.22-7.59 (m, 4H), 6.81-7.91 (m, 4H), $5.0(\mathrm{~s}, 1 \mathrm{H}), 3.6$ (s, 2H), 3.21 (t, $J=7.5 \mathrm{~Hz}, 4 \mathrm{H}), 2.27$ (t, $J=7.6 \mathrm{~Hz}$, $4 \mathrm{H}), 2.23$ (s, 3H); ${ }^{13} \mathrm{C} \mathrm{NMR}\left(400 \mathrm{MHz}, \mathrm{CDCl}_{3}\right) \delta \mathrm{ppm}$ : $45.1\left(\mathrm{CH}_{3}\right), 47.0\left(\mathrm{CH}_{2}\right.$, diazepine $) 48.9\left(\mathrm{CH}_{2}\right.$, piperazine), 113.2, 155.0 (C, phenoxyl), 115.9, $122.1(\mathrm{CH}$, benzimidazole), 116.2, $127.3(\mathrm{CH}$, phenoxyl), 126.8, $132.7(\mathrm{CH}$, arene), 130.0, 148.1 (C, arene), 142.3 (C, benzimidazole), $152.4(\mathrm{C}=\mathrm{N}$, benzimidazole $), 158.7$ $(\mathrm{C}=\mathrm{O}), 164.2(\mathrm{C}=\mathrm{N}, \mathrm{C}-\mathrm{O})$; $\mathrm{MS}:\left[\mathrm{M}^{+}\right]$: 478, Anal. Calcd. for $\mathrm{C}_{28} \mathrm{H}_{26} \mathrm{~N}_{6} \mathrm{O}_{2}: \mathrm{C}, 70.28 ; \mathrm{H}, 5.48 ; \mathrm{N}, 17.56$; Found: C, 70.16; H, 5.50; N, 17.61.

\subsection{Biological studies}

Condensed heterocyclic systems containing oxadiazole, pyrimidine, imidazole, and benzimidazole and 1,4-benzodiazepines have attracted the attention of chemists owing to these nuclei having been identified in the literature as the most active pharmacophores in drug design and synthesis. It has been observed that incorporation of certain bioactive pharmacophores in the existing drug molecules sometimes exert a profound influence on the biological profiles of that molecule. All the compounds were screened for their anti-microbial activity by disc diffusion method at $100 \mu \mathrm{g} / \mathrm{mL}$ concentration in DMF against Pseudomonas aeruginosa (MTCC 1688) and Bacillus cerus (MTCC 1305) and anti-fungal activity against Macrophomina phaseolina (MTCC 166) and Fusarium solani (MTCC 350). The zone of inhibition and activity index were determined in comparison of the standard drugs 'Streptomyacin' and 'fluconazol'. The outcome of this study is presented in tabular form in table 1. All these compounds were found to be active against the bacterial and fungal strains.

\section{Results and Discussion}

Ubiquity of 1,4-benzodiazepine nucleus in chemical literature is undoubtedly a consequence of multifarious 
Table 1. Anti-bacterial and antifungal activity of compounds 7-14.

\begin{tabular}{|c|c|c|c|c|c|c|c|c|}
\hline \multirow[b]{2}{*}{ Comp. No. } & \multicolumn{2}{|c|}{$\begin{array}{l}\text { Pseudomonas } \\
\text { aeruginosa }\end{array}$} & \multicolumn{2}{|c|}{ Bacillus cerus } & \multicolumn{2}{|c|}{$\begin{array}{c}\text { Macrophomina } \\
\text { phaseolina }\end{array}$} & \multicolumn{2}{|c|}{ Fusarium solani } \\
\hline & $\begin{array}{l}\text { Zone of } \\
\text { inhibition }\end{array}$ & $\begin{array}{c}\% \text { activity } \\
\text { Compared } \\
\text { to the } \\
\text { standard }\end{array}$ & $\begin{array}{l}\text { Zone of } \\
\text { inhibition }\end{array}$ & $\begin{array}{l}\% \text { activity } \\
\text { Compared } \\
\text { to the } \\
\text { standard }\end{array}$ & $\begin{array}{l}\text { Zone of } \\
\text { inhibition }\end{array}$ & $\begin{array}{c}\% \text { activity } \\
\text { Compared } \\
\text { to the } \\
\text { standard }\end{array}$ & $\begin{array}{l}\text { Zone of } \\
\text { inhibition }\end{array}$ & $\begin{array}{c}\% \text { activity } \\
\text { Compared } \\
\text { to the } \\
\text { standard }\end{array}$ \\
\hline $\begin{array}{l}\text { Streptomycin } \\
\text { (std. Antibacterial) }\end{array}$ & 25 & 100 & 24 & 100 & - & - & - & - \\
\hline $\begin{array}{l}\text { Flucanazol } \\
\text { (std. Anti-fungal) }\end{array}$ & - & - & - & - & 26 & 100 & 26 & 100 \\
\hline 7 & 18 & 72.0 & 23 & 95.8 & 24 & 92.3 & 20.3 & 78.0 \\
\hline 8 & 22 & 88.0 & 22 & 91.6 & 21.3 & 81.9 & 15.1 & 58.0 \\
\hline 9 & 15 & 60.0 & 21 & 87.5 & 24 & 92.3 & 12.3 & 47.3 \\
\hline 10 & 15.1 & 60.4 & 18 & 75.0 & 24.5 & 94.2 & 13.2 & 50.7 \\
\hline 11 & 24 & 96.0 & 19 & 79.1 & 25 & 96.1 & 15.0 & 57.6 \\
\hline 13 & 23 & 92.0 & 22.5 & 93.7 & 23 & 88.4 & 23.6 & 90.7 \\
\hline 14 & 24.5 & 98.0 & 20 & 83.3 & 22 & 84.6 & 20.2 & 77.6 \\
\hline
\end{tabular}

biological response which they elicit in combating a variety of body ailments. Recent demonstrations that some of their derivatives can be used as privileged templates in the development of potential agents for the treatment of Acquired Immunodeficiency Syndrome (AIDS) ${ }^{16}$ has stimulated further interest in this nucleus from yet another perspective. As a part of our endeavour to create novel heterocyclic scaffolds of anticipated biological activity from easily accessible starting materials, here in this paper we report, the preliminary results of our studies on the synthesis of oxadiazole, pyrimidine, imidazole, and benzimidazole incorporated 1,4-benzodiazepines linked to it through a 2-p-phenoxyl spacer in 10-14. Compound (7) was obtained through a five step strategy on isatin (1). The first step of this strategy involved the conversion of $N$-chloroacetylisatin (2) to 1,4-benzodiazepine-2one-5-carboxylate (3), using a literature procedure. ${ }^{17-20}$ In view of the medicinal importance of piperazine derivatives, it was considered of interest to append this nucleus on to the 5-methoxycarbonyl function to form the 5-carboxamido substituted derivatives (4), which in the subsequent step was treated with $\mathrm{POCl}_{3}{ }^{21}$ in dimethylaniline (DMA) to afford the corresponding 2-chloro derivative (5). The 2-Cl atom (an imino chloride/imidoyl chloride) is a highly reactive species known to be activated for nucleophillic attack. Its nucleophillic displacement with $p$-hydroxybenzonitrile (6) yielded compound 7 (scheme 1). ${ }^{22-24}$ A perusal of literature $^{25}$ on the potential of amidines and imidate ester intermediates for their use as versatile precursors in synthesis, has demonstrated that these were readily formed from the reaction of compound 7 with
$\mathrm{H}_{2} \mathrm{~N}-\mathrm{OH} . \mathrm{HCl}+\mathrm{KOH}$ (in $\mathrm{MeOH}$ ) following the procedure reported in the literature ${ }^{26}$ for such reactions on substrates containing the nitrile group. Established protocols on amidine derivative (8) was applied, employing the reagents acetyl chloride and dimethylacetylene dicarboxylate to form the compounds (10) (oxadiazole bearing) and (11) (pyrimidine bearing) benzodiazepine nucleus respectively (scheme 1 ). The second key intermediate was (9) formed from the reaction of compound 7 with $\mathrm{R}-\mathrm{OH}+\mathrm{HCl}$. Established protocols on imidate derivative (9) was applied, employing the reagents ethylenediamine and $o$-phenylenediamine to form the dihydroimidazole (12) [whose oxidation with iodoxybenzoic acid provided imidazole (13)] and benzimidazole (14) bearing benzodiazepine nucleus, respectively (scheme 2). ${ }^{27-30}$ All the synthesized compounds gave satisfactory results of their microanalysis, IR, ${ }^{1} \mathrm{H}$ NMR, ${ }^{13} \mathrm{C}$ NMR and MS spectral data which were found to be consistent to the assigned structures.

\section{Conclusion}

In conclusion, two elegant protocols have been developed to provide an easy access to the biologically active novel oxadiazole, pyrimidine, imidazole and benzimidazole incorporated analogues of 1,4-benzodiazepine derivatives linked to it by a phenoxyl spacer, utilizing the potential of corresponding amidine (8) and imidate (9) intermediates in high yield and purity. The formulated plan provided an easy incorporation of the vital fragments of the biologically active nuclei and the anti-HIV prone 1,4-benzodiazepine nuclei together, in 
the same molecular framework in acceptable yield and purity, for the evaluation of their bio-efficiency over the existing potential antimicrobial agents.

\section{Supporting Information}

Copies of ${ }^{1} \mathrm{H}$ NMR, ${ }^{13} \mathrm{C}$ NMR spectra, mass spectra, analytical reports and representative photographs of the zone of inhibition is provided in the supporting information at www.ias.ac.in/chemsci.

\section{Acknowledgement}

Authors are thankful to Punjab University, Chandigarh for providing the spectral data of the compounds. Authors are also thankful to Department of Science and Technology (DST), New Delhi for financial support provided to 'Banasthali Centre for Education and Research in Basic Sciences' under their Consolidation of University Research for Innovation and Excellence in Women Universities (CURIE) programme.

\section{References}

1. Evans B E, Rittle K E, Bock M G, DiPardo R M, Freidinger R M, Whitter W L, Lundell G F, Veber D F, Anderson P S, Chang R S L, Lotti V J, Cerino D J, Chen T B, Kling P J, Kunkel K A, Springer J P and Hirshfieldt J 1988 J. Med. Chem. 312235

2. Horton D A, Bourne G T and Smythe M L 2003 Chem. Rev. $\mathbf{1 0 3} 893$

3. Poupaert J, Carato P, Colacino E and Yous S 2005 Curr. Med. Chem. 12877

4. Triggle D 2003 Cell Mol. Neurobiol. 23293

5. De Simone R W, Currie K S, Mitchell S A, Darrow J W and Pippin D A 2004 Comb. Chem. High Throughput Screening 7473

6. Patchett A A and Nargund R P 2000 Аnпи. Rep. Med. Chem. 35289

7. Pathak V N, Joshi R and Gupta N 2007 Indian. J. Chem. Sect., B 461191

8. Bariwal J B, Upadhayay K D and Manvar A T 2008 Eur. J. Med. Chem. 432279

9. Summa V, Petrocchi A, Bonelli F, Crescenzi B, Donghi M, Ferrara M, Fiore F, Gardelli C, Paz G O, Hazuda J D,
Jones P, Kinzel O, Laufer R, Monteagudo E, Muraglia E, Nizi E, Orvieto F, Pace P, Pescatore G, Scarpelli R, Kara S, Marc V W and Michael R 2008 J. Med. Chem. 515843

10. Zareef M, Iqbal R, Al-Masoudi N A, Zaidi J H, Arfan M and Shahzad S A 2007 Synth. Commun. 182281

11. Nofal Z M, Fahmy H H, Zarea E S and EI-Eraky W 2011 Acta Pol. Pharm. Drug Res. 68507

12. Jyoti P, Vinod T K, Shyam V S, Vinita C, Bhatnagar S, Sinha S, Gaikwad A N and Tripathi R P 2009 Eur. J. Med. Chem. 443350

13. Guven O O, Erdogan T, Goeker H and Yildiz S 2007 Bioorg. Med. Chem. Lett. 172233

14. Chavan N L, Naik N H, Nayak S K and Kusurkar R S 2010 ARKIVOC 248

15. Leiby R W 1985 J. Org. Chem. 502926

16. Shashikant J, Golak C M, Shyam T, Ashok C and Mukund K G 2010 Org. Process Res. Dev. 14657

17. Ogata M and Matsumoto H 1976 Chem. Ind. (London) 1067

18. Sharma P, Vashistha B, Tyagi R, Srivastava V, Shorey M, Singh B and Kishore D 2010 Int. J. Chem. Sci. 841

19. Singh A, Sirohi R, Shastri S and Kishore D 2003 Ind. J. Chem. 42B 3124

20. Santra P K, Kishore D and Jain P 2003 Synth. Commun. 333695

21. Karthikeyan M S, Prasad D J, Mahalinga M, Holla B S and Kumari N S 2008 Eur. J. Med. Chem. 4325

22. Rashid M, Ahmad B and Mishra R. 2010 Int. J. Pharm. Sci. 2616

23. Cortes E C, Franco R S and Mellado O G $2001 \mathrm{~J}$. Heterocycl. Chem. 38663

24. Del G M R, Gatta F, Pandolfi C and Settimj G 1982 Farmaco 37343

25. Bekircan O and Bektas H 2008 Molecules 132126

26. Pace P, Emila M, Francesso D, Gardelli C, Harper S, Muraglia E, Nizi E, Orvieto F, Petroocchi A, Poma M, Rowely M, Scarpelli R, Laufer R, Paz O G, Monteagudo E, Bonelli F, Hazuda D, Stillmock K A and Summa V 2007 J. Med. Chem. 502225

27. Summa V, Petrocchi A, Matassa V G, Taliani M, Laufer R, Francesco R, Altamura S and Pace P 2004 J. Med. Chem. 475336

28. Zhong Y, Zhou H, Gauthier D R and Askin D 2006 Tetrahedron Lett. 471315

29. Bellomo A, Celebi-Olcum N, Bu X, Rivera N, Ruck R T, Welch C J, Houk K N and Dreher S D 2012 Angew. Chem. Int. Ed. $\mathbf{5 1} 6912$

30. Vincent A B, John M, Parimala H, Jeanine P, Charles J R and Thomas R W 2009 J. Comb. Chem. 111100 